\title{
Metabolomics and Cardiology: Toward the Path of Perinatal Programming and Personalized Medicine
}

\author{
Roberta Pintus, ${ }^{1}$ Pier Paolo Bassareo, ${ }^{2}$ Angelica Dessì, ${ }^{1}$ Martino Deidda, \\ Giuseppe Mercuro, ${ }^{2}$ and Vassilios Fanos ${ }^{1}$ \\ ${ }^{1}$ Department of Surgery, Neonatal Intensive Care Unit, Neonatal Pathology and Neonatal Section, University of Cagliari, \\ Policlinico Universitario, Strada Statale 554, Km 4.500, Bivio di Sestu, Monserrato, 09042 Cagliari, Italy \\ ${ }^{2}$ Department of Medical Sciences "M. Aresu”, Unit of Cardiology and Angiology, University of Cagliari, Policlinico Universitario, \\ Strada Statale 554, Km 4.500, Bivio di Sestu, Monserrato, 09042 Cagliari, Italy
}

Correspondence should be addressed to Angelica Dessì; angelicadessi@hotmail.it

Received 3 February 2017; Revised 15 May 2017; Accepted 28 May 2017; Published 3 July 2017

Academic Editor: Peter J. Oefner

Copyright (C) 2017 Roberta Pintus et al. This is an open access article distributed under the Creative Commons Attribution License, which permits unrestricted use, distribution, and reproduction in any medium, provided the original work is properly cited.

\begin{abstract}
Heart diseases are one of the leading causes of death in Western Countries and tend to become chronic, lowering the quality of life of the patients and ending up in a massive cost for the Health Systems and the society. Thus, there is a growing interest in finding new technologies that would allow the physician to effectively treat and prevent cardiac illnesses. Metabolomics is one of the new "omics" sciences enabling creation of a photograph of the metabolic state of an individual exposed to different environmental factors and pathologies. This review analyzed the most recent literature about this technology and its application in cardiology in order to understand the metabolic shifts that occur even before the manifestation of these pathologies to find possible early predictive biomarkers. In this way, it could be possible to find better treatments, ameliorate the patient's quality of life, and lower the death rate. This technology seems to be so promising that several industries are trying to set up kits to immediately assess the metabolites variations in order to provide a faster diagnosis and the best treatment specific for that patient, offering a further step toward the path of the development of a tailored medicine.
\end{abstract}

\section{Introduction}

Cardiac pathologies are a critical health issue affecting millions of people worldwide with a constant mortality rate in particular in the elderly, a difficult prognosis, and a worsening in quality life of affected people. In fact, they tend to become chronic and lead to several complications that may affect other vital organs such as brain, lungs, and kidneys. Indeed cardiovascular diseases (CVDs) are globally the number one cause of death: more people die every year from CVDs than from any other cause (17.5 million deaths, an estimation of $31 \%$ of all deaths worldwide). People with cardiovascular pathologies or who are at high cardiovascular risk need early detection since the $80 \%$ of premature heart diseases are preventable [1].

Among the complications of these pathologies there are pulmonary edema or respiratory tract infections, kidney insufficiency, and stroke. In children, cardiovascular diseases or congenital heart malformations can lead to pulmonary hypertension and neurodevelopmental problems due to the lack of oxygen supply [2].

The pathophysiology of heart pathologies is complex. Indeed, recent findings pointed out a possible pivotal role of mitochondrial dysfunction and the subsequent altered energy metabolism in cardiac diseases, in particular in case of heart failure [3].

In general, patient management could be quite challenging and demanding; thus there is a need for the clinician to have the best tools that can improve and facilitate the diagnosis and the prognosis for these diseases.

\section{Metabolomics: The Skeleton Key of Cardiac Diseases?}

During the last decade, animal and human studies have applied metabolomics to cardiovascular research, using both 


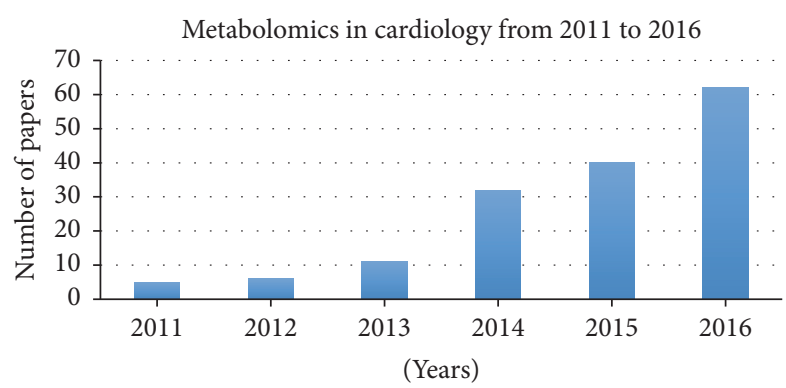

FIGURe 1: PubMed results concerning the studies of metabolomics and cardiology from 2011 to 2016.

targeted and untargeted approaches; as such, metabolic fingerprints have been identified for several cardiovascular risk factors and diseases [4].

Indeed, by entering the keywords "metabolomics" and "cardiology" on PubMed, this will show 161 papers, 156 written from 2011 to 2016 (Figure 1).

In fact metabolomics is a new technique that allows investigators to study the metabolic network involved in heart diseases so as to better understand their pathophysiological mechanism. Griffin et al. highlighted how the classical metabolomics technique could be applied in cardiology; indeed high resolution Magnetic Resonance Imaging (MRI) and mass spectrometry (MS) are extremely useful for gaining information about cardiac disease processes since they are both highly discriminant for a range of pathological processes starting from cardiac ischemia (angina and myocardial infarction) to heart failure [5]. These techniques could be applied to both heart tissue and biofluids such as blood and saliva with a minimum compliance needed from the patient, since their collection is not invasive. Metabolomics is one of the newest "omics" science and before its broad application, scientists tried to investigate the metabolic variation in both physiological and pathological states using proteomics or transcriptomics, but these techniques have several limitations; for instance, they are not "real-time" meaning that in case of disease occurrence the modification in the proteome or transcriptome modifications are much slower than modifications in the metabolome [6].

In Table 1 the most relevant studies are reported concerning metabolomics in cardiology on PubMed from 2011 to 2016. There are 18 studies, involving 3.874 patients in total: 2351 suffered from acute cardiac pathologies, while 736 suffered from chronic heart illnesses and 786 were controls. Most studies (9) performed ${ }^{1} \mathrm{H}-\mathrm{NMR}$ analysis. The prevalent biofluid analyzed was plasma (10 studies) followed by serum (7 studies), urine (3), and breath (1).

In 2011 Kang et al. investigated metabolomics urinary profiles of elderly patients with ischemic heart failure, using ${ }^{1} \mathrm{H}$-nuclear magnetic resonance $\left({ }^{1} \mathrm{H}\right.$-NMR) [23]. The patients compared to controls showed different levels of ketonic bodies as a marker of altered myocardial metabolism meaning that one of the pathological features of this pathology could be a reduction in fatty acids oxidation and an increase of glucose metabolism.

Among others, the study performed by Desmoulin et al. in 2013 underlines the predictive power of metabolomics
[19]. It is a prospective study on a cohort of acute heart failure patients admitted in the cardiac intensive care unit and it assessed survival at 30 days. The plasma was collected on admission. They found out that lactate and cholesterol were the discriminating metabolites predicting 30-day mortality; in particular patient with high lactate and low cholesterol on admission showed increased mortality. This lactate/cholesterol rate in plasma could be a useful and simple parameter to apply in clinical practice in order for the physician to make the best decision in heart failure care.

Another interesting study performed by Deidda et al. in 2015 questioned whether there could be any changes in patients metabolome according to the worsening of their conditions [4]. They compared blood samples of patients affected by mild to moderate impairment of left ventricle ejection fraction and of others affected by severe left ventricle ejection fraction impairment and controls. After the statistical analysis, they identified 3 metabolic clusters related to the 3 groups. The responsible metabolites specific for each heart failure stage are 2-hydroxybutyrate, glycine, methylmalonate, and myoinositol and they might reflect both an increase in energy demand and an impaired ability to generate ATP (see also Table 1). The fingerprint identification of a still-free-ofsymptoms myocardial impairment, which directly correlates with the more sensitive echocardiographic parameters of myocardial contractility, could enable a better monitoring of at-risk individuals, allowing the anticipation of systolic function worsening and/or the development of an episode of overt failure.

In line with presented data, a latest review published in the Journal of the American College of Cardiology stated that metabolomics is transforming the ability to predict, identify, and better understand several cardiac diseases, by allowing monitoring of the effectiveness of therapeutic interventions, thus leading to advancing the objective of personalizing the practice of medicine [24].

\section{Perinatal Programming and Cardiology}

The perinatal programming of adult diseases ( $\mathrm{DOHaD}$ theory) states that every adverse event that may occur during pregnancy "shapes" the health status of the fetus and its development and could affects its life course [25]. Thus this theory emphasizes the importance of this delicate period of life in which everything must be timed properly in order to avoid future complications such as cardiac diseases in adolescence and adult life.

In fact Bassareo et al. investigated the cardiac outcome of young adults born with extremely low birth weight (ELBW) [26]. At 25 years of age they are at risk of major cardiac consequences such as sudden death due to the prolongation in QT interval or they are at higher risk of hypertension due to the reduced brachial-flow mediated vasodilatation compared to those born appropriate for gestational age (see also Table 2) (Bassareo PP, "Long Term Problems in Young Adults Born ELBW” [26-30]).

It is therefore a big challenge, on the opposite side of the life span, to try to predict the cardiac outcome of the neonate during pregnancy. Metabolomics seems to have made it 


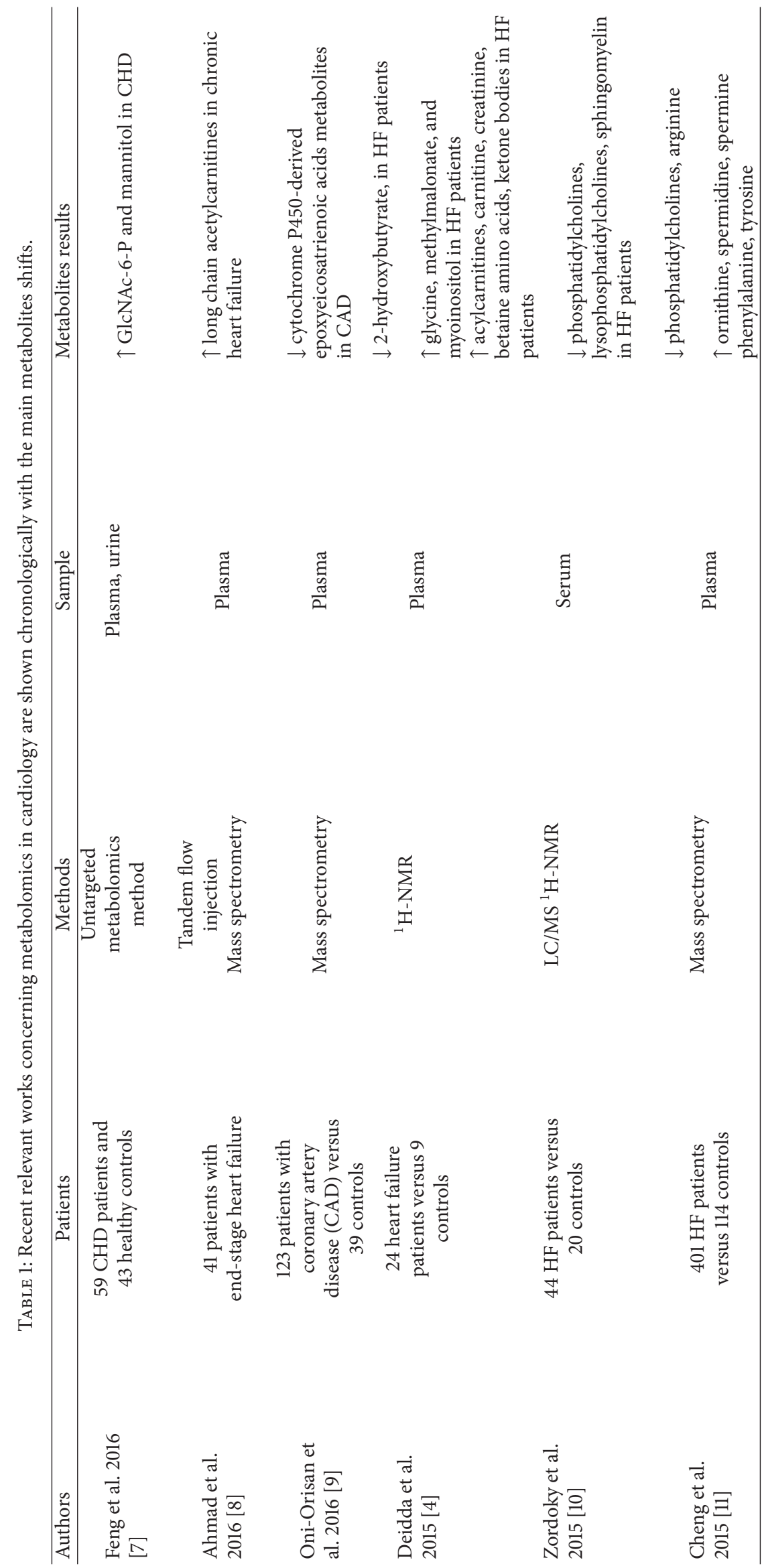




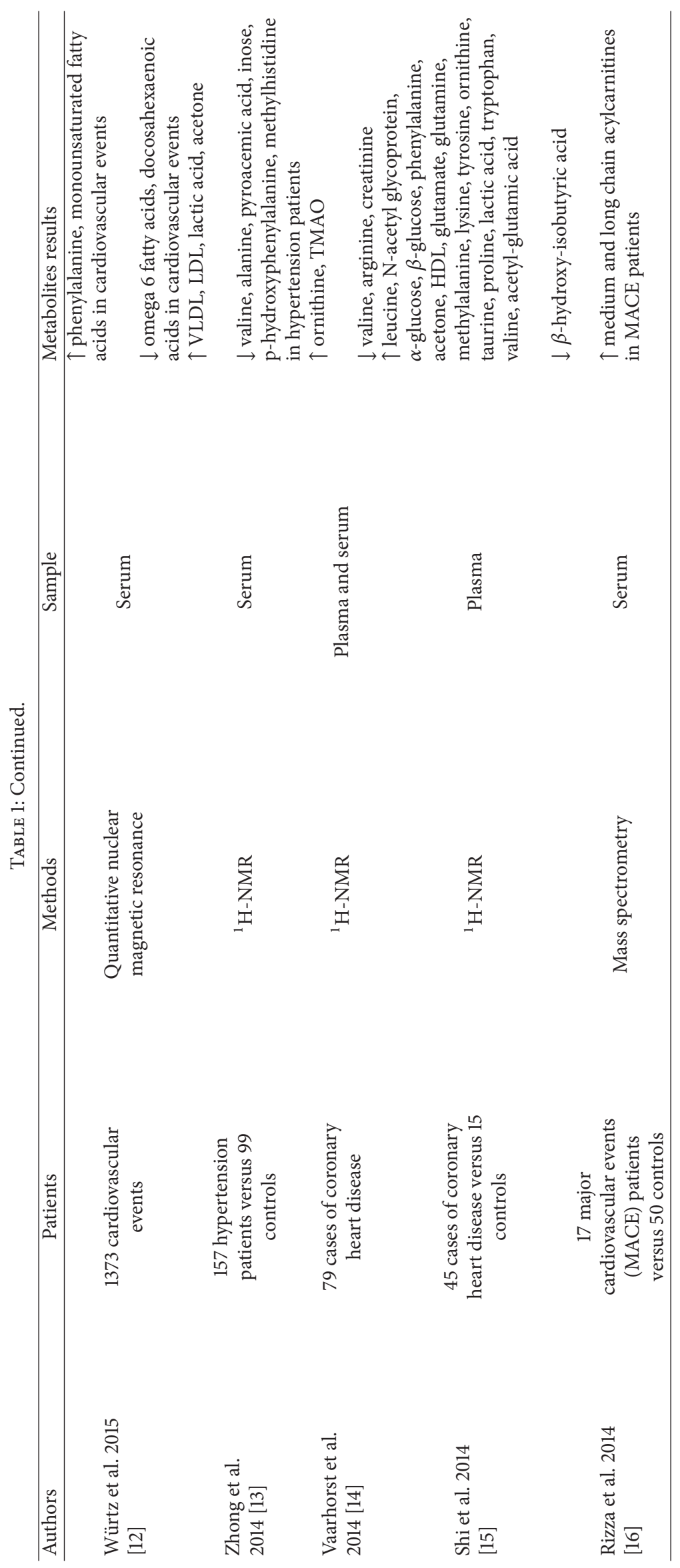




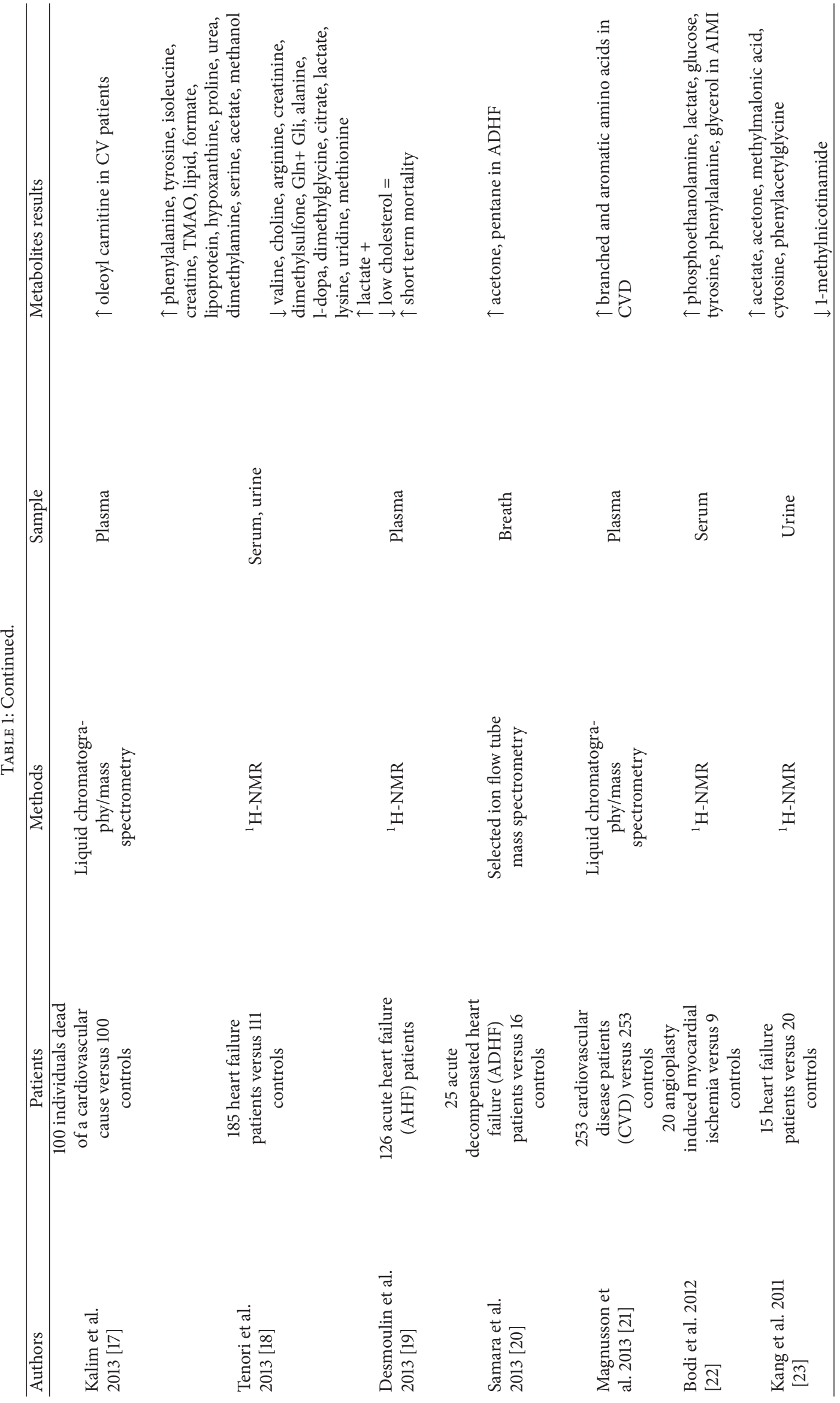


TABLE 2: Possible long-term consequences in adulthood to subjects born with extremely low birth weight: suggestion for diagnosis and care.

\begin{tabular}{|c|c|c|}
\hline $\begin{array}{l}\text { Possible consequences in } \\
\text { adulthood }\end{array}$ & Risks & Suggestion for diagnosis and care \\
\hline $\begin{array}{l}\text { Increase in the QT interval } \\
\text { of ECG in some subjects }\end{array}$ & $\begin{array}{l}\text { Risk of arrhythmia and } \\
\text { sudden death }\end{array}$ & $\begin{array}{l}\text { ECG monitoring } \\
\text { Avoidance of drugs that increase the } \\
\text { QT interval }\end{array}$ \\
\hline Reduced vascular elasticity & Risk of hypertension & Blood pressure monitoring \\
\hline High ADMA levels & $\begin{array}{l}\text { Risk of acute cardiovascular } \\
\text { problems }\end{array}$ & ECG and blood pressure monitoring \\
\hline $\begin{array}{l}\text { Increase in } \\
\text { microalbuminuria and } \\
\text { urinary NGAL, reduction } \\
\text { of kidney volume }\end{array}$ & $\begin{array}{l}\text { Risk of chronic kidney } \\
\text { insufficiency }\end{array}$ & $\begin{array}{l}\text { Urine stick monitoring, albuminuria, } \\
\text { creatinine, and cystatin C in the blood, } \\
\text { kidney ultrasound }\end{array}$ \\
\hline
\end{tabular}

possible even though to our knowledge there is only one study of metabolomics in pregnancy performed by Bahado-Singh et al. [31]. Their aim was to identify metabolomics markers in maternal first-trimester serum for the detection of fetal congenital heart defect. Serum from mothers of CVD fetuses showed a significant disturbance in acylcarnitine and sphingomyelin and other metabolites related to an abnormal lipid metabolism. These findings may help the future development of devices to be used at the bedside similar to those that are already being sold to assess the cardiac troponin in patients with suspected acute coronary syndrome by just a finger prick of blood [32].

Moving from neonates to infants and young adults (since CVDs have a long latent period), metabolomics could be a useful tool to investigate the actual role of genetic predisposition. It could help understand whether a particular gene mutation is protective or harmful and in which metabolism it is involved or if there are any sex differences. A very interesting study concerning this topic was performed by Klein et al. in 2014 [33]. They studied the SORT1 gene through genomics and metabolomics and were able to determine several effects of the mutations of this gene in young males and females.

\section{Metabolomics Cross Talks with Microbiomics Even in the Occurrence of Heart Diseases?}

Metabolomics allows not only measuring changes in metabolites concentrations, but also discriminating those of human origin from those of microbial origins; in fact several authors consider this technology as the Rosetta Stone of microbiomics [34]. Indeed there are 3 types of metabolites in humans: those of human origins (produced only by eukaryotic cells); those of microbial origins (produced only by prokaryotic cells); and those of common origins [35]. Among the most studied ones is hippurate which both is a marker of renal function and can be produced by gut microbiota as well [36]. Nevertheless, in most cases, the origin of the molecules might not be unambiguously determined by using only metabolomics, but this technique is unique in its possibility of simultaneously analyzing molecules from both host and microbes in a single measurement. In a review of 2013 written by Russell et al. they highlighted the different fates of choline for the first time [37]. This metabolite, involved in the methionine-betaine-choline cycle, if metabolized by an altered microbiota, is transformed into trimethylamine $\mathrm{N}$ oxide (TMAO). It is found in several peripheral tissues but in particular in the arterial epithelium in case of atherosclerosis. At the beginning of this year, a very interesting study was performed by Feng et al. in which the integrated metabolomics and metagenomics analysis of plasma and urine allowed identifying metabolites of microbial origins in coronary heart disease [7]. These molecules, mannitol and N-acetyl-D-glucosamine-6-phosphate among others, are related to a particular strand of Clostridium spp. or Streptococcus spp., indicating a possible role of the dysbiosis in the occurrence of this pathology. In November 2016 a review written by Jonsson and Bäckhed was published, concerning all the recent literature about the role of gut microbiota in atherosclerosis [38]. They stated that specific strand of bacteria, Proteobacteria, can be found in the atherosclerotic plaque. This phylum comprises the genera of Helicobacter and Chryseomonas and it is the most abundant in the plaque. Actinobacteria can be found in the plaque as well, while in patients with coronary artery disease (CAD) the number of Bacteroidetes decreases compared to controls and the ratio of Firmicutes/Bacteroidetes increases. They also proposed 3 possible mechanisms by which microbiota could affect the development of atherosclerotic plaque.

The first one: bacterial infection activates the immune system causing an excessive inflammatory response that may turn out to be dangerous, independently of the site of invasion. The subsequent proatherogenic response could be mediated by Toll-like receptor 4 expressed in macrophages.

The second: the TMAO production could initiate the activation of platelets and foam cells.

The third: the production of noxious molecules such as the previously mentioned TMAO is related to the diet and gut microbiota metabolites.

With metabolomics it is possible to demonstrate that apparently healthy young adults who were born with birth weight $<1000 \mathrm{~g}$ present a specific profile compared to apparently healthy young adults who were born at term. That is perinatal programming. 
Differences in the two groups were related to the alterations in the arginine and proline metabolism, in the purine and pyrimidine metabolism in the histidine, in beta-alanine metabolism, and in the urea cycle [39].

\section{Future Perspectives}

The most investigated cardiac pathologies are heart failure, coronary heart disease, and myocardial infarction.

Several studies displayed an alteration of metabolites concerning lipid metabolism, highlighting the energy imbalance as a peculiar feature of such pathologies.

On the other hand, some authors showed different metabolites that indicate an interaction between diet and microbiota.

These findings open up unusual scenarios to the cardiologist and although it is normal to feel some sort of incredulity, they could pave the way to new possibilities of early diagnosis and individualized treatment. Congenital malformations, gut colonization by microbiota, individual genetic arrangement, and its interplay with both behavioral and risk factors, such as drugs assumption, can influence the occurrence of heart diseases. Metabolomics, for its peculiarities, seems to be the most promising technology to investigate the individual predisposition or the eventual long-term prognosis of these pathologies.

\section{Conflicts of Interest}

The authors declare that there are no conflicts of interest regarding the publication of this paper.

\section{References}

[1] WHO sites about Cardiovascular diseases available on line from September 2016, http://www.who.org/.

[2] P. P. Bassareo, L. Saba, P. Solla, C. Barbanti, A. R. Marras, and G. Mercuro, "Factors influencing adaptation and performance at physical exercise in complex congenital heart diseases after surgical repair," BioMed Research International, vol. 2014, Article ID 862372, 2014.

[3] D. A. Brown, J. B. Perry, M. E. Allen et al., "Expert consensus document: Mitochondrial function as a therapeutic target in heart failure," Nature Reviews Cardiology, vol. 14, no. 4, pp. 238250, 2016.

[4] M. Deidda, C. Piras, C. C. Dessalvi et al., "Metabolomic approach to profile functional and metabolic changes in heart failure," Journal of Translational Medicine, vol. 13, no. 1, article 297, 2015.

[5] J. L. Griffin, H. Atherton, J. Shockcor, and L. Atzori, "Metabolomics as a tool for cardiac research," Nature Reviews Cardiology, vol. 8, no. 11, pp. 630-643, 2011.

[6] D. I. Ellis, W. B. Dunn, J. L. Griffin, J. W. Allwood, and R. Goodacre, "Metabolic fingerprinting as a diagnostic tool," Pharmacogenomics, vol. 8, no. 9, pp. 1243-1266, 2007.

[7] Q. Feng, Z. Liu, S. Zhong et al., "Integrated metabolomics and metagenomics analysis of plasma and urine identified microbial metabolites associated with coronary heart disease," Scientific Reports, vol. 6, Article ID 22525, 2016.
[8] T. Ahmad, J. P. Kelly, R. W. McGarrah et al., "Prognostic implications of long-chain acylcarnitines in heart failure and reversibility with mechanical circulatory support," Journal of the American College of Cardiology, vol. 67, no. 3, pp. 291-299, 2016.

[9] A. Oni-Orisan, M. L. Edin, J. A. Lee et al., "Cytochrome P450derived epoxyeicosatrienoic acids and coronary artery disease in humans: a targeted metabolomics study," Journal of Lipid Research, vol. 57, no. 1, pp. 109-119, 2016.

[10] B. N. Zordoky, M. M. Sung, J. Ezekowitz et al., "Metabolomic fingerprint of heart failure with preserved ejection fraction," PLoS ONE, vol. 10, no. 5, Article ID e0124844, 2015.

[11] M.-L. Cheng, C.-H. Wang, M.-S. Shiao et al., "Metabolic disturbances identified in plasma are associated with outcomes in patients with heart failure: Diagnostic and prognostic value of metabolomics," Journal of the American College of Cardiology, vol. 65, no. 15, pp. 1509-1520, 2015.

[12] P. Würtz, A. S. Havulinna, P. Soininen et al., "Metabolite profiling and cardiovascular event risk: a prospective study of 3 population-based cohorts," Circulation, vol. 131, no. 9, pp. 774$785,2015$.

[13] L. Zhong, J.-P. Zhang, A.-G. Nuermaimaiti, and K.-X. Yunusi, "Study on plasmatic metabolomics of Uygur patients with essential hypertension based on nuclear magnetic resonance technique," European Review for Medical and Pharmacological Sciences, vol. 18, no. 23, pp. 3673-3680, 2014.

[14] A. A. M. Vaarhorst, A. Verhoeven, C. M. Weller et al., "A metabolomic profile is associated with the risk of incident coronary heart disease," American Heart Journal, vol. 168, no. 1, pp. 45-e7, 2014.

[15] Q. Shi, H. Zhao, and J. Chen, "Study on Qi deficiency syndrome identification modes of coronary heart disease based on metabolomic biomarkers," Evidence-Based Complementary and Alternative Medicine, vol. 2014, Article ID 281829, 15 pages, 2014.

[16] S. Rizza, M. Copetti, C. Rossi et al., "Metabolomics signature improves the prediction of cardiovascular events in elderly subjects," Atherosclerosis, vol. 232, no. 2, pp. 260-264, 2014.

[17] S. Kalim, C. B. Clish, J. Wenger et al., "A plasma long-chain acylcarnitine predicts cardiovascular mortality in incident dialysis patients," Journal of the American Heart Association, vol. 2, no. 6, Article ID e000542, 2013.

[18] L. Tenori, X. Hu, P. Pantaleo et al., "Metabolomic fingerprint of heart failure in humans: a nuclear magnetic resonance spectroscopy analysis," International Journal of Cardiology, vol. 168, no. 4, pp. e113-e115, 2013.

[19] F. Desmoulin, M. Galinier, C. Trouillet et al., "Metabonomics Analysis of Plasma Reveals the Lactate to Cholesterol Ratio as an Independent Prognostic Factor of Short-Term Mortality in Acute Heart Failure," PLoS ONE, vol. 8, no. 4, Article ID e60737, 2013.

[20] M. A. Samara, W. H. W. Tang, F. Cikach Jr. et al., "Single exhaled breath metabolomic analysis identifies unique breathprint in patients with acute decompensated heart failure," Journal of the American College of Cardiology, vol. 61, no. 13, pp. 1463-1464, 2013.

[21] M. Magnusson, G. D. Lewis, U. Ericson et al., "A diabetespredictive amino acid score and future cardiovascular disease," European Heart Journal, vol. 34, no. 26, pp. 1982-1989, 2013.

[22] V. Bodi, J. Sanchis, J. M. Morales et al., "Metabolomic profile of human myocardial ischemia by nuclear magnetic resonance spectroscopy of peripheral blood serum: a translational study based on transient coronary occlusion models," Journal of the 
American College of Cardiology, vol. 59, no. 18, pp. 1629-1641, 2012.

[23] S.-M. Kang, J.-C. Park, M.-J. Shin et al., "1H nuclear magnetic resonance based metabolic urinary profiling of patients with ischemic heart failure," Clinical Biochemistry, vol. 44, no. 4, pp. 293-299, 2011.

[24] J. R. Ussher, S. Elmariah, R. E. Gerszten, and J. R. B. Dyck, “The Emerging Role of Metabolomics in the Diagnosis and Prognosis of Cardiovascular Disease," Journal of the American College of Cardiology, vol. 68, no. 25, pp. 2850-2870, 2016.

[25] D. J. P. Barker, "The developmental origins of well-being," Philosophical Transactions of the Royal Society B: Biological Sciences, vol. 359, no. 1449, pp. 1359-1366, 2004.

[26] P. P. Bassareo, M. Puddu, G. Flore et al., "Could ADMA levels in young adults born preterm predict an early endothelial dysfunction?" International Journal of Cardiology, vol. 159, no. 3, pp. 217-219, 2012.

[27] P. P. Bassareo, V. Fanos, M. Puddu, C. Cadeddu, M. Balzarini, and G. Mercuro, "Significant QT interval prolongation and long QT in young adult ex-preterm newborns with extremely low birth weight," Journal of Maternal-Fetal and Neonatal Medicine, vol. 24, no. 9, pp. 115-118, 2011.

[28] P. P. Bassareo, V. Fanos, M. Puddu et al., "Reduced brachial flow-mediated vasodilation in young adult ex extremely low birth weight preterm: A condition predictive of increased cardiovascular risk?" Journal of Maternal-Fetal and Neonatal Medicine, vol. 23, no. 3, pp. 121-124, 2010.

[29] P. P. Bassareo, V. Fanos, M. Mussap et al., "Urinary NGAL and hematic ADMA levels: An early sign of cardio-renal syndrome in young adults born preterm?" Journal of Maternal-Fetal and Neonatal Medicine, vol. 26, no. 2, pp. 80-83, 2013.

[30] P. P. Bassareo, A. R. Marras, L. Cugusi, A. M. Zedda, and G. Mercuro, "The reasons why cardiologists should consider prematurity at birth and intrauterine growth retardation among risk factors," Journal of Cardiovascular Medicine, vol. 17, no. 5, pp. 323-329, 2016.

[31] R. O. Bahado-Singh, R. Ertl, R. Mandal et al., "Metabolomic prediction of fetal congenital heart defect in the first trimester," American Journal of Obstetrics and Gynecology, vol. 211, no. 3, pp. 240.el-240.e14, 2014.

[32] D. W. Kemper, V. Semjonow, F. de Theije et al., "Analytical evaluation of a new point of care system for measuring cardiac Troponin I," Clinical Biochemistry, vol. 50, no. 4-5, pp. 174-180, 2017.

[33] M. S. Klein, K. E. Connors, J. Shearer, H. J. Vogel, and D. S. Hittel, "Metabolomics reveals the sex-specific effects of the sort1 low-density lipoprotein cholesterol locus in healthy young adults," Journal of Proteome Research, vol. 13, no. 11, pp. 50635070, 2014.

[34] G. Sharon, N. Garg, J. Debelius, R. Knight, P. C. Dorrestein, and S. K. Mazmanian, "Specialized metabolites from the microbiome in health and disease," Cell Metabolism, vol. 20, no. 5, pp. 719-730, 2014.

[35] K. B. Martinez, V. Leone, and E. B. Chang, "Microbial metabolites in health and disease: Navigating the unknown in search of function," Journal of Biological Chemistry, vol. 292, no. 21, pp. 8553-8559, 2017.

[36] L. Guoa, M. V. Milburna, J. A. Ryalsa et al., "Plasma metabolomic profiles enhance precision medicine for volunteers of normal health," Proceedings of the National Academy of Sciences of the United States of America, vol. 112, no. 35, pp. E4901-E4910, 2015.
[37] W. R. Russell, L. Hoyles, H. J. Flint, and M.-E. Dumas, "Colonic bacterial metabolites and human health," Current Opinion in Microbiology, vol. 16, no. 3, pp. 246-254, 2013.

[38] A. L. Jonsson and F. Bäckhed, "Role of gut microbiota in atherosclerosis," Nature Reviews Cardiology, vol. 14, no. 2, pp. 79-87, 2016.

[39] V. Fanos, L. Barberini, R. Antonucci, and L. Atzori, "Metabolomics in neonatology and pediatrics," Clinical Biochemistry, vol. 44, no. 7, pp. 452-454, 2011. 


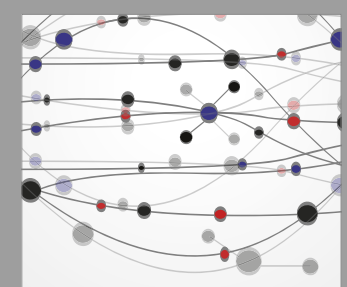

The Scientific World Journal
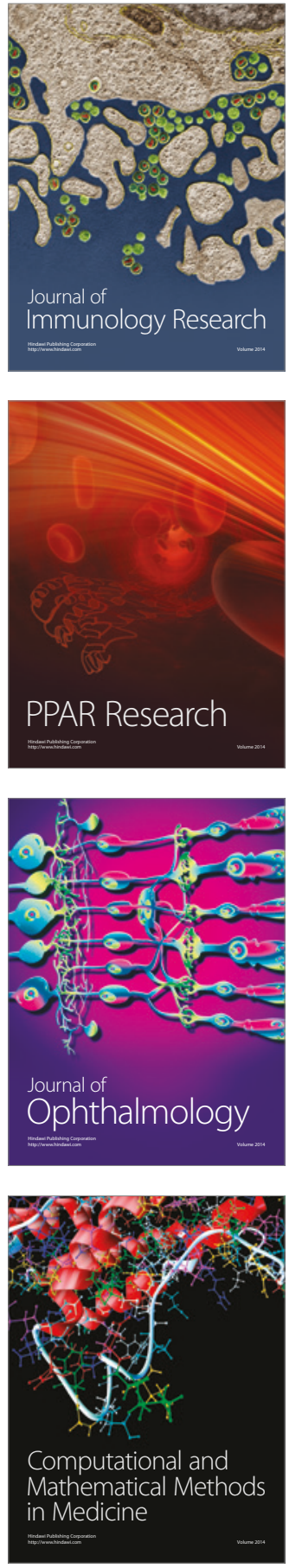

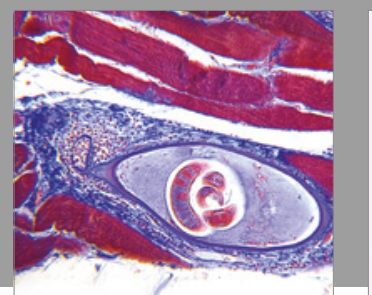

Gastroenterology Research and Practice
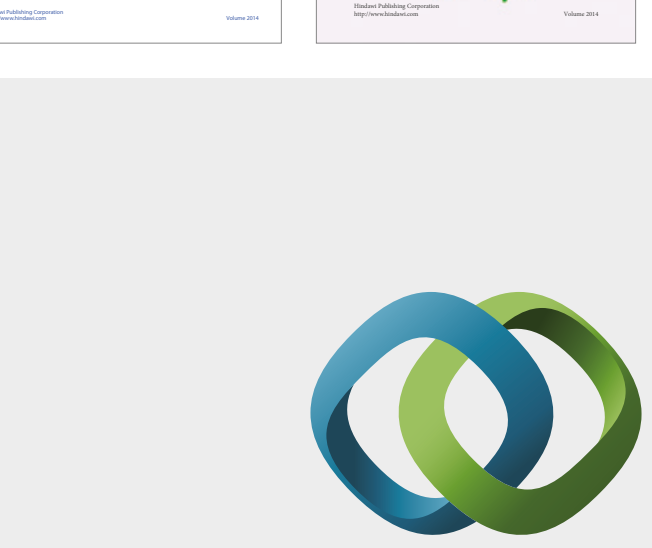

\section{Hindawi}

Submit your manuscripts at

https://www.hindawi.com
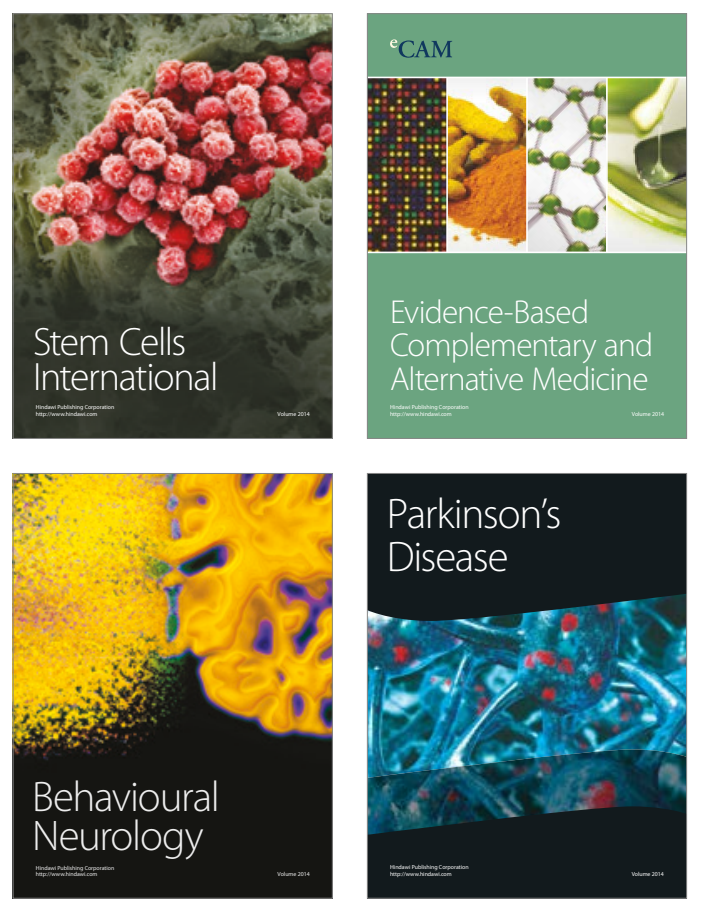
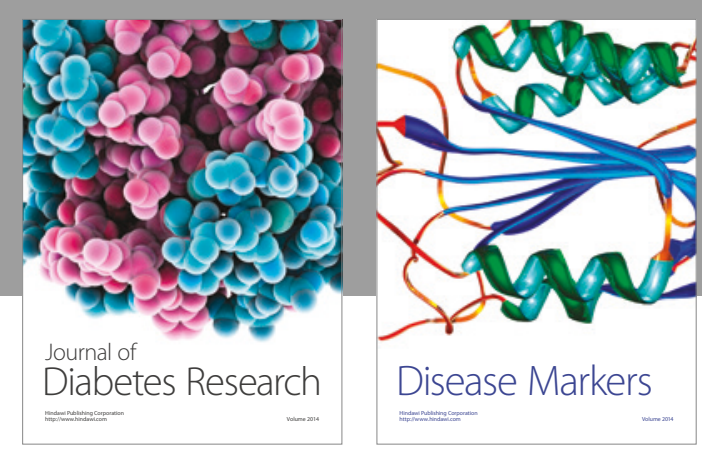

Disease Markers
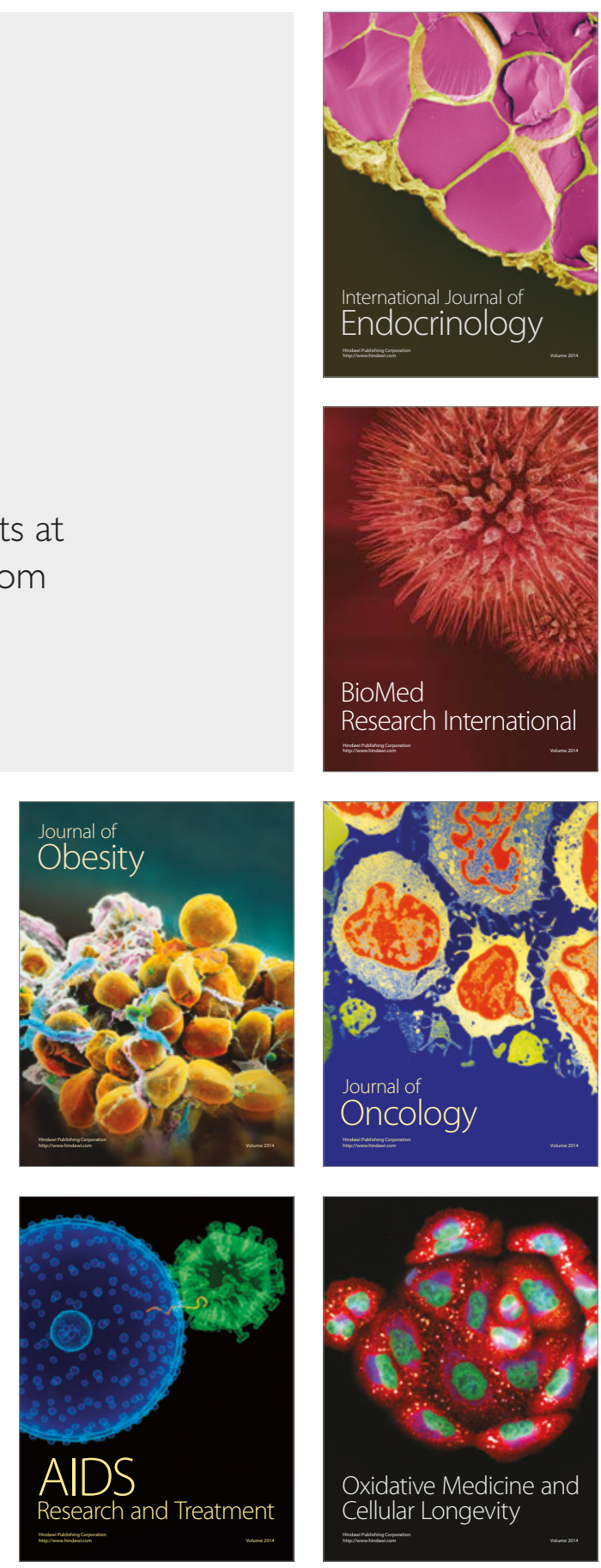